\title{
Condições de trabalho e processos de adoecimento: impactos na saúde do profissional do serviço social no campo da saúde
}

Working condlitions and illness processes: impacts on the health of social service professionals in the health field

Condiciones de trabajo y procesos de enfermedad: impactos en la salud de los profesionales de los servicios sociales en el campo de la salud

Kaio Germano Sousa da Silva ORCID: https://orcid.org/0000-0003-4236-6230

Faculdade do Vale Elvira Dayrell, Brasil E-mail: kaiogsds@hotmail.com

Samara da Conceição Ferreira Guimarães

ORCID: https://orcid.org/0000-0003-3772-8225 Centro Universitário de Ciências e Tecnologia do Maranhão, Brasil

E-mail: samaraferreira10@outlook.com

Elaine Ferreira do Nascimento ORCID: https://orcid.org/0000-0002-1632-9148

Fundação Oswaldo Cruz, Brasil

E-mail: negraelaine@gmail.com

Francioneide Patricia de Sousa

ORCID: https://orcid.org/0000-0002-1482-0013

Faculdade Adelmar Rosado, Brasil

E-mail: francioneidepatricia@gmail.com

Cristina Soares Oliveira

ORCID: https://orcid.org/0000-0002-4917-6811

Centro Universitário de Ciências e Tecnologia do Maranhão, Brasil

E-mail: csoaresoliveira27@gmail.com

Luana Pereira Ibiapina Coêlho

ORCID: https://orcid.org/0000-0002-2054-959X

Universidade Estadual do Maranhão, Brasil

E-mail: luana_ibiapina@ hotmail.com

Eduardo Brito da Silva

ORCID: https://orcid.org/0000-0002-8571-7806

Centro Universitário de Ciências e Tecnologia do Maranhão, Brasil

E-mail: eduzinhobds@gmail.com

Eudilene da Silva Mesquita

ORCID: https://orcid.org/0000-0001-9894-0903

Centro Universitário de Ciências e Tecnologia do Maranhão, Brasil

E-mail: eu.mesquitta@gmail.com

Franklane Silva Cantanhede

ORCID: https://orcid.org/0000-0001-5115-4817

Centro Universitário de Ciências e Tecnologia do Maranhão, Brasil

E-mail: cfranklane@gmail.com

George do Carmo Leão

ORCID: https://orcid.org/0000-0001-9106-1500

Centro Universitário de Ciências e Tecnologia do Maranhão, Brasil

E-mail: georgeleao13@gmail.com

Isadora Sayonara Ferreira Coelho

ORCID: https://orcid.org/0000-0002-3300-6244

Centro Universitário de Ciências e Tecnologia do Maranhão, Brasil

E-mail: isaaebella3006@gmail.com

Ruth Lany Barros Araújo

ORCID: https://orcid.org/0000-0003-4981-1165

Centro Universitário de Ciências e Tecnologia do Maranhão, Brasil

E-mail: ruthlany@hotmail.com

Thalita Cristina Oliveira Santos

ORCID: https://orcid.org/0000-0002-4284-7328

Centro Universitário de Ciências e Tecnologia do Maranhão, Brasil E-mail: thalitacristina181@gmail.com 
Victória Ribeiro de Sousa Marques

ORCID: https://orcid.org/0000-0002-6512-1212

Centro Universitário de Ciências e Tecnologia do Maranhão, Brasil

E-mail: marquesvick24@gmail.com

Raquel Ferreira Coelho

ORCID: https://orcid.org/0000-0003-2988-544X

Universidade Federal do Piaú, Brasil

E-mail: raquelxcoelho@hotmail.com

Kayle Rodrigues dos Santos

ORCID: https://orcid.org/0000-0001-8343-5621

Centro Universitário de Ciências e Tecnologia do Maranhão, Brasil

E-mail: kaylepsicologo18@gmail.com

Alessandra Ferreira das Chagas

ORCID: https://orcid.org/0000-0002-2407-0666

Centro Universitário de Ciências e Tecnologia do Maranhão, Brasil

E-mail: 18alenzo@gmail.com

Ana Cristina Jácome Castelo Gomes

ORCID: https://orcid.org/0000-0002-3098-2872

Centro Universitário de Ciências e Tecnologia do Maranhão, Brasil

E-mail: anajacomebb4@gmail.com

Milene Sousa Carvalho

ORCID: https://orcid.org/0000-0003-3993-1032

Centro Universitário de Ciências e Tecnologia do Maranhão, Brasil

E-mail: Millenanegocios@outlook.com

Mélrica Mariana Andrade Alves

ORCID: https://orcid.org/0000-0002-5944-0022

Centro Universitário de Ciências e Tecnologia do Maranhão, Brasil

E-mail: mmariana531@gmail.com

Washington Walber Macedo dos Santos

ORCID: https://orcid.org/0000-0003-4577-1143

Centro Universitário de Ciências e Tecnologia do Maranhão, Brasil

E-mail: mwashingtonwalber@gmail.com

Rosinei Nascimento Ferreira

ORCID: https://orcid.org/0000-0003-2732-7778

Instituição de Gestão Educacional Signorelli, Brasil

E-mail: rosineifff2@hotmail.com

Antônia Keila Alves da Costa

ORCID: https://orcid.org/0000-0002-0917-0108

Centro Universitário de Ciências e Tecnologia do Maranhão, Brasil

E-mail: keylla_costa@outlook.com

Mayra Ramalho de Sousa Rêgo

ORCID: https://orcid.org/0000-0003-4704-8645

Centro Universitário de Ciências e Tecnologia do Maranhão, Brasil

E-mail: mayraramalhoofc3@gmail.com

Rafael Roberval Teixeira Silva

ORCID: https://orcid.org/0000-0001-5683-5769

Centro Universitário Maurício de Nassau, Brasil

E-mail: rafael.apolonioteixeira@gmail.com

Livia Graciela Machado de Oliveira

ORCID: https://orcid.org/0000-0002-5680-0985

Universidade Estadual do Maranhão, Brasil

E-mail: liviamachado535@gmail.com

Flavia Gomes de Oliveira

ORCID: https://orcid.org/0000-0003-2652-2176

Escola Nacional de Saúde Pública Sergio Arouca, Brasil

E-mail: flaviagomesofc@gmail.com

Laellya Larhanna Lima Santos Gonçalves

ORCID: https://orcid.org/0000-0002-4753-5593

Centro Universitário de Ciências e Tecnologia do Maranhão, Brasil

E-mail: laellyalima@gmail.com

Mogeane Pereira Lopes Antunes

ORCID: https://orcid.org/0000-0002-5524-6265

Centro Universitário Maurício de Nassau, Brasil

E-mail: mogeanem12@gmail.com

Tâmara Valeria Nery de Santana

ORCID: https://orcid.org/0000-0002-6593-0722

Faculdade de Minas Fabriciano, Brasil

E-mail: Tamara_nery@hotmail.com 


\begin{abstract}
Resumo
A pesquisa teve como objetivo analisar as principais causas do adoecimento sócio ocupacional do Assistente Social. Onde o estudo trata-se de uma pesquisa qualitativa que utilizou uma análise bibliográfica de caráter narrativo, que foi realizada através de consulta em bases de dados online de pesquisa, sendo elas: Scielo (Scientific Eletronic Library OnLine), BVS (Biblioteca Virtual da Saúde), e Google acadêmico. O contexto atual exige demais dos profissionais e os coloca frequentemente em riscos de adoecimento. Onde a formação continuada ajuda o profissional a pensar em respostas efetivas para sua proteção para múltiplas expressões da questão social, tal formação deve ser dever do estado para melhorar no atendimento público. Deve-se destacar ainda que, a atuação dos assistentes sociais pode compreender qualquer uma das etapas de vida de uma pessoa. Neste aspecto, durante o luto, o profissional pode buscar formas de ajudar o individuo a vivenciar a experiência sem comprometer sua vida de forma permanente. Quando o trabalhador adoece não afeta somente ele, existe uma rede de pessoas acometidas pelo um trabalhador doente onde se pode dizer que é a família, pois a mesma vive diariamente com o trabalhador. Por fim, o estudo permitiu aprofundar os conhecimentos acerca da saúde do trabalhador, da assistência social e os fatores que influenciam o adoecimento dos profissionais que atuam na área. Espera-se que mais pesquisas sejam realizadas nesta linha de investigação a fim de comprovar a importância destes profissionais e a necessidade de prestar assistência a saúde física e psicológica dos mesmos.
\end{abstract}

Palavras-chave: Condições de trabalho; Assistentes sociais; Capitalismo.

\begin{abstract}
The research aimed to analyze the main causes of socio-occupational illness of the Social Worker. Where the study is a qualitative research that used a bibliographic analysis of a narrative character, which was carried out by consulting online research databases, namely: Scielo (Scientific Electronic Library OnLine), VHL (Virtual Health Library), and academic Google. The current context demands too much from professionals and often puts them at risk of illness. Where continuing education helps professionals to think of effective responses for their protection to multiple expressions of the social issue, such training should be a duty of the state to improve public service. It should also be noted that the role of social workers can include any of the stages of a person's life. In this aspect, during mourning, the professional can look for ways to help the individual to live the experience without compromising their life permanently. When the worker gets sick, it doesn't only affect him, there is a network of people affected by a sick worker where it can be said that it is the family, as it lives daily with the worker. Finally, the study allowed to deepen the knowledge about workers' health, social assistance and the factors that influence the illness of professionals working in the area. It is expected that more research will be carried out in this line of investigation in order to prove the importance of these professionals and the need to provide assistance to their physical and psychological health.
\end{abstract}

Keywords: Working conditions; Social workers; Capitalism.

\title{
Resumen
}

La investigación tuvo como objetivo analizar las principales causas de enfermedad sociolaboral del Trabajador Social. Donde el estudio es una investigación cualitativa que utilizó un análisis bibliográfico de carácter narrativo, el cual se realizó mediante la consulta de bases de datos de investigación en línea, a saber: Scielo (Biblioteca Electrónica Científica OnLine), BVS (Biblioteca Virtual en Salud) y Google académico. El contexto actual exige demasiado de los profesionales y, a menudo, los pone en riesgo de enfermarse. Donde la educación continua ayuda a los profesionales a pensar en respuestas efectivas para su protección a múltiples expresiones del problema social, dicha capacitación debe ser un deber del Estado para mejorar el servicio público. También debe tenerse en cuenta que el papel de los trabajadores sociales puede incluir cualquiera de las etapas de la vida de una persona. En este aspecto, durante el duelo, el profesional puede buscar formas de ayudar al individuo a vivir la experiencia sin comprometer su vida de forma permanente. Cuando el trabajador se enferma no solo le afecta, existe una red de personas afectadas por un trabajador enfermo donde se puede decir que es la familia, ya que convive a diario con el trabajador. Finalmente, el estudio permitió profundizar en el conocimiento sobre la salud de los trabajadores, la asistencia social y los factores que influyen en la enfermedad de los profesionales que laboran en el área. Se espera que se realicen más investigaciones en esta línea de investigación con el fin de demostrar la importancia de estos profesionales y la necesidad de brindar asistencia a su salud física y psicológica.

Palabras clave: Condiciones de trabajo; Trabajadores sociales; Capitalismo.

\section{Introdução}

Segundo Antunes e Luci (2015) em seu estudo traz à tona o capitalismo feroz que é um fator determinante para o surgimento de um conceito denominado "a sociedade dos doentes de trabalho", o capitalismo contemporâneo com ênfase monopolista não liga o bem-estar do trabalhador somente para o lucro onde a acumulação capitalista começou a ser mais lucrativa no período de transição do século XX ao século XXI, onde a modificação está dentro do trabalho seja ela nos locais, na organização e na competição. 
Para a realização deste estudo trabalhou-se uma revisão de literatura de cunho narrativo, baseada em uma ampla discussão da produção temático-científica, que teve como ponto de partida responder o seguinte questionamento: Quais aspectos influenciam no processo de adoecimento do Assistente Social no seu trabalho?

Para obter tal resposta tiveram-se como base os seguintes objetivos: Analisar as principais causas do adoecimento sócio ocupacional do Assistente Social, de forma mais específica, buscou-se: discutir as principais doenças que acomete o profissional Assistente Social no espaço de trabalho devido ao capitalismo contemporâneo; interpretar as estratégias para o profissional assistente social lidar com o sofrimento no trabalho; e descrever as atribuições e competências do Assistente Social na saúde.

\section{Metodologia}

O estudo trata-se de uma pesquisa qualitativa que utilizou uma análise bibliográfica de caráter narrativo, que foi realizada através de consulta em bases de dados online de pesquisa, sendo elas: SciELO (Scientific Eletronic Library OnLine), BVS (Biblioteca Virtual da Saúde), e Google acadêmico (Silva et al., 2021).

Como critérios de inclusão, foram selecionados estudos em português, inglês e espanhol que estavam com textos completos e que abordavam a temática proposta, durante os anos de 2009 a 2021. Para o critério de exclusão foi realizada uma breve leitura dos resumos e títulos dos respectivos artigos e textos selecionados e após o entendimento de todo o assunto foram excluídos aqueles que não tratavam do assunto proposto.

Posteriormente procuraram-se explicações para os resultados diferentes ou conflitantes nos diferentes estudos, e ainda foram buscados dados que pudessem direcionar ao estudo de condições de trabalho e processo de adoecimento no campo do Assistente Social e aspectos que influenciam tais ações, visando elaborar e fortalecer as estratégias da saúde pública e social contra doenças relacionadas às más condições de trabalho.

Em seguida foram discutidos os principais resultados das pesquisas, através de uma avaliação crítica dos estudos incluídos, comparando-os com os conhecimentos teóricos, identificando conclusões e implicações resultantes. Por fim, constou a síntese dos conhecimentos e elaboração da discussão da revisão, com as conclusões da pesquisa.

\section{Resultados e Discussão}

A necessidade de conhecer quais as causas que provocam o adoecimento do profissional assistente social no seu processo de trabalho no campo da saúde é de suma importância para conhecer as principais doenças relacionadas a seu trabalhar no e com resultados buscar fortalecimento de estratégias da saúde pública e melhorias de trabalho tanto no contexto físico e social contra doenças relacionadas aos hábitos alimentares não saudáveis e diminuição de incidência das mesmas, já que os hábitos alimentares na adolescência podem ser mudados com apoio da família, escola e ajuda de profissionais.

Em uma análise detalhada dos artigos selecionados para a realização deste estudo traz-se à tona um conceito reflexivo que parte de três pilares principais deste trabalho, são eles: capitalismo feroz, precarização do trabalho e o Assistente Social como profissional adoecido, e esses três pontos norteiam o tema: Condições de trabalho e processos de adoecimento: os impactos na saúde do profissional no campo da saúde.

$\mathrm{Na}$ busca de compreender e dar resolutividade à questão social vê-se o profissional Assistente Social lutando contra o capitalismo monopolizado, más condições de trabalho, baixos salários que contribuíram fortemente para o processo de adoecimento e saúde, pois tais fatores agem diretamente neste processo (Kato \& Assumpção, 2009; Maurício, Carvalho, \& Carmo, 2017).

Trazendo assim na perspectiva do trabalho delimitado que debate e discute o tema supracitado acima se verifica nas palavras de Guiraldelli (2010), o qual fala sobre condições de trabalho do Assistente Social, ele foca na formação continuada 
para melhorar a qualidade do atendimento. Deve-se ressaltar também a importância de dispositivos celulares para facilitar a comunicação entre os profissionais e os clientes (Miranda et al., 2019).

Um dos requisitos básicos é a necessidade da formação continuada para garantirmos competência e qualidade na atuação profissional cotidiana de forma a pensarmos respostas efetivas para as múltiplas manifestações da questão social. A partir dos anos 1990, verifica-se um adensamento das pesquisas e da literatura no campo do Serviço Social que se aproxima de forma rigorosa do pensamento crítico-dialético com respaldo na tradição marxista. Desde então, a profissão passa por um processo de maturação e reconhecimento no campo da produção de conhecimento, trazendo contribuições significativas para a formação e o exercício profissional (Guiraldelli, 2010).

O contexto atual exige demais dos profissionais e os coloca frequentemente em riscos de adoecimento (Melo et al., 2021). Onde a formação continuada ajuda o profissional a pensar em respostas efetivas para sua proteção para múltiplas expressões da questão social, tal formação deve ser dever do estado para melhorar no atendimento público.

Deve-se destacar ainda que, a atuação dos assistentes sociais pode compreender qualquer uma das etapas de vida de uma pessoa. Neste aspecto, durante o luto, o profissional pode buscar formas de ajudar o individuo a vivenciar a experiência sem comprometer sua vida de forma permanente (Soares et al., 2021; Cruz et al., 2021).

Quando o trabalhador adoece não afeta somente ele, existe uma rede de pessoas acometidas pelo um trabalhador doente onde se pode dizer que é a família, pois a mesma vive diariamente com o trabalhador (Torres, Chagas, Moreira, Barreto, \& Rodrigues, 2011). Ainda em Torres as sequelas de um trabalhador adoecido são muitas e preocupantes, a limitação funcional, alterações psicoafetivas, perda da autoestima e isolamento social onde as mesmas são causadas no contexto do trabalho, mercado competitivo (a incerteza de trabalho), más condições de trabalho e salários baixos.

O Assistente Social como inserido na classe trabalhadora vive diariamente com a questão social, precarização de trabalho e baixos salários, tais fatores contribuem fortemente para o seu adoecimento, neste ponto, a pandemia dificultou sua atuação e acrescentou maior risco potencial (Melo et al., 2021). O processo de adoecimento do Assistente Social está diretamente ligado aos fatores citados. Ainda traz considerações importantes das condições de trabalho e o processo de adoecimento do Assistente Social (Câmara \& Oliveira, 2013).

$\mathrm{O}$ acidente e as doenças relacionadas ao trabalho constituem-se um sério problema social e de saúde pública que atinge um número significativo de trabalhadores. A combinação das inovações tecnológicas como os novos métodos gerenciais, tem gerado intensificação do trabalho traduzida numa série de agravos físicos e mentais à saúde. Enquanto trabalhador (a) assalariado(a), a (o) Assistente Social não está isento (a) desta implicação, uma vez que o trabalho é determinante nos modos de viver e de trabalhar dos sujeitos e, nesse sentido, o acidente e o adoecimento são expressões que retratam a maneira como o trabalho vem se configurando na atualidade na vida dos trabalhadores (Câmara \& Oliveira, 2013).

Como se pode observar no estudo da autora o Assistente Social como trabalhador assalariado não é uma exceção do capitalismo, está fortemente influenciado pelas modernizações tecnológicas, pelo mercado competitivo, dentre outros fatores, pois no âmbito do trabalho existe uma rede de situações tanto interna como externa que influencia tanto o Assistente Social e seus usuários.

Com isso se debate a importância da inserção do Assistente Social na saúde para nortear os doentes do trabalho a fim de contribuir com a recuperação deste doente mostrando as causas internas e externas provocadas pela alienação capitalista, o debate da inserção do Assistente Social como cuidar do trabalhador como problemas da questão social começou nas grandes manifestações populares da reforma sanitária (Nogueira \& Sarreta, 2009).

Na perspectiva do Assistente Social como trabalhador assalariado observa-se que o mesmo sofre com violação de direitos, configuração inadequada de seus espaços sócio-ocupacionais e são submetidos a aceitar determinadas condições de trabalho para não deixar seus usuários sem atendimento, onde os impactos dessas ações são de longo prazo na vida do 
Assistente Social podendo causar LER/DORT (Raichelis, 2009; Da Silva \& Ramos, 2015).

A precarização do trabalho e o desmonte dos direitos conquistados pela classe trabalhadora vêm sofrendo imensas transformações devido à capitalização moderna e a alienação da tecnologia, as más condições de trabalho interferem diretamente no trabalho e na vontade de trabalhar, já a precarização do trabalho contribui para a baixa autoestima, com isso traz novas formas de trabalho moldando um perfil suscetível a doenças (Jesus, 2017; Mauricio et al., 2010).

Segundo Ceolin (2014) a precarização do trabalho se dá na crise capitalista, pois quando o capitalismo entra em crise ele se torna mais agressivo se adaptando para se preocupar somente em ganhos com poucas perdas, obrigando o trabalhador a trabalhar sem cessar para mostrar os ganhos ao empregador com a necessidade de mostrar trabalho e se fazer necessário. Compreendendo Mauricio et al. (2010) a partir de Jesus (2017) pode-se fazer uma análise profunda de doenças que acometem a classe trabalhadora devido ao capitalismo que vive em crise, são elas: a baixa autoestima por muitas vezes não conseguir acompanhar o mercado agressivo, a depressão causada pelo sentimento de incapacidade, LER/DORT, hipertensão devido a pressões que o trabalhador sofre, entre outras doenças.

O Assistente Social como profissional inserido na classe trabalhadora sofre com os mesmos processos dos demais trabalhadores suscetíveis também a serem acometidos por doenças devido a essas transformações do capitalismo cruel motivado pela ganância da elite. Deste modo, Da Silva e Ramos (2015) destaca o desafio dos trabalhadores e do Serviço Social na luta, demonstra esse sistema monopolista a fim de manter seus direitos já conquistados e a busca de novos direitos, já o Assistente Social é um profissional que garante qualidade de vida.

Nessa direção, o trabalho do Assistente Social realiza-se na contramão do projeto societário vigente. Os Assistentes Sociais tecem seu cotidiano de trabalho numa relação tensionada entre a defesa dos direitos sociais e as premissas da sociedade capitalista, cuja intervenção se dá diretamente na contradição capital-trabalho (Faermann \& Melo, 2016).

O assistente social está submetido a tensões onde se embasam na contramão de seu trabalho de como deve ser feito tomando como obrigação e adaptação ao modelo capitalista obrigado a trabalhar em contradições, tornando seu cotidiano de trabalho exaustivo e estressante, com jornadas de trabalho longas e exaustivas às más condições de trabalho. $\mathrm{O}$ assistente social se torna vulnerável ao processo de adoecimento já que o mesmo está ligado ao combate das mazelas sociais. Faermann e Melo (2016) em seu estudo destaca as principais doenças que acometem os Assistentes Sociais, patologias de cunho mental, como depressão, ansiedade, baixa autoestima física, hipertensão, ansiedade e doenças ocupacionais.

Entretanto, a importância de a categoria lutar em prol de melhorias de trabalho é imensamente importante, pois quando se trabalha em um local onde não se adoece por conta das precarizações, faz toda a diferença na vida do trabalhador que faz parte da classe trabalhadora, é importante destacar a implementação de discussões acerca da precarização do trabalho dos Assistentes Sociais, pois a literatura que trata desses assuntos é precária.

Enfim, é de suma importância que o profissional Assistente Social analise de forma crítica a realidade para não incorporar a prática que possa lhe prejudicar como trabalhador e usuário e impor seus direitos para a melhoria de qualidade de vida em um ambiente de trabalho respaldado na ética a fim de incentivar a relação profissional, usuário e empregador saudável (Iamamoto, 2012).

\section{Considerações Finais}

Partindo dos dados obtidos constatou-se que a saúde do (a) trabalhador (a), é uma área que precisa ter maior visibilidade nas formas de como esse trabalho é desenvolvido e no local onde esse profissional desenvolve suas técnicas laborais. Essa discussão pode promover e estimular os assistentes sociais que buscam aprender sobre o contexto laboral no qual estão inseridos, pois, ao relacionar suas vivências no trabalho com as transformações que vêm ocorrendo neste âmbito, estas afetam diretamente na vida e nas suas condições de trabalho, pois o mesmo, por questão de sobrevivência, vende sua força de 
trabalho. Em muitos campos de trabalho do assistente social, em que o mesmo pode ser inserido, sabe-se das muitas dificuldades que esse profissional presencia e vive durante o exercício de sua prática e que diariamente os seus direitos têm sido violados.

Por fim, o estudo permitiu aprofundar os conhecimentos acerca da saúde do trabalhador, da assistência social e os fatores que influenciam o adoecimento dos profissionais que atuam na área. Espera-se que mais pesquisas sejam realizadas nesta linha de investigação a fim de comprovar a importância destes profissionais e a necessidade de prestar assistência a saúde física e psicológica dos mesmos.

\section{Referências}

Antunes, R., \& Luci, P. (2015). A sociedade dos adoecimentos no trabalho. Serv. Soc. Soc., (123), 407-427.

Armstrong, T. (2001). Inteligências Múltiplas na sala de aula (2a ed.). Artes Médicas.

Câmara, P. C. S., \& Oliveira, I. M. (2013). As condições de trabalho na área de saúde e o processo de adoecimento do (a) assistente social (Dissertação de mestrado). Univerisdade Federal do Rio Grande do Norte, Natal, RN, Brasil.

Ceolin, G. F. (2014). Crise do capital, precarização do trabalho e impactos no Serviço Social. Serv. Soc. Soc, 5(8), $239-264$.

Cruz, M. C. N. L., Gonçalves, F. T. D., Araújo, Z. A. M., Dutra, G. C., Vaz, A. C., Oliveira, A. T. F., \& Melo, K. C. (2021). Um pedaço de mim virou estrelinha: elaboração do luto infantil. Research, Society and Development. 10(8), e23210817255.

Da Silva, K. L., Ramos, S. R. (2015). Desafios da classe trabalhadora: Serviço Social na luta por condições de trabalho. Revista em Pauta, 13(36), 197-223.

Faermann, L. A., \& Melo, C. C. V. As condições de trabalho dos assistentes sociais e suas implicações no processo de adoecimento dos profissionais. Textos \& Contextos, 15, 96-113.

Iamamoto, M. V. (2012). O serviço social na contemporaneidade: trabalho e formação profissional. Cortez.

Jesus, A. S. P. N. (2017). Entre a dor e a alegria - a invisibilidade do adoecimento dosas assistentes sociais no município de Cachoeira-BA (Trabalho de Conclusão de Curso). Universidade Federal Do Recôncavo Da Bahia, Cachoeira, BA, Brasil.

Kato, L. S., \& Assumpção, P. F. S. (2009). O serviço social nos processos de licença por motivo de doença em pessoa da família: o cuidar do outro e o cuidar de si (Trabalho de Conclusão de Curso). Universidade Federal De Santa Catarina, Florianópolis, SC, Brasil.

Maurício, J. M. M., Carvalho, M. C., \& Carmo, R. C. D. (2017). Adoecimento no trabalho e sofrimento social: um estudo preliminar sobre os impactos na saúde do assistente social. Viii Jornada Internacional de Políticas Públicas, 2010(8), 01-07.

Melo, K. C., Da Silva, C. O., Souza, M. W. S. S., Soares, A. N., Da Silva, W. C., Oliveira, F. B. M., \& Silva, R. A. D. (2021). Síndrome de burnout em profissionais da saúde que atuam na linha de frente da Covid-19. International Journal of Development Research, 11(02), 44706-44711.

Mendes, K. D. S., Silveira, R. C. C. P., \& Galvão, C. M. (2008). Revisão integrativa: método de pesquisa para a incorporação de evidências na saúde e na enfermagem. Texto contexto - enferm, 17(4), 758-764.

Miranda, L. S. C., Costa, L. S. D., Mota, D. S., Da Silva, W. C., Melo, K. C., Soares, A. N., \& Santos, M. S. (2019). Dispositivos celulares como ferramenta de comunicação entre o profissional da saúde e o cliente, Revista Eletrônica Acervo Saúde, 36, e1689.

Nogueira, D. O., \& Sarreta, F. (2009). A inserção do assistente social na saúde: desafios atuais. Anais do Simpósio Mineiro de Assistentes Sociais, Belo Horizonte, MG, Brasil, 4.

Raichelis, R. (2009). O assistente social como trabalhador assalariado: desafios frente a violação de seus direitos. Serviço Social e Sociedade, (107), 420-437.

Silva, K. G. S., Magalhaes, M. J. S., Pessoa, N. M., Morais, E. R. O., Lima, A. S. S., Santos, M. E. L. D. C., \& Silva, L. A. S. (2021). Perfil alimentar de adolescentes no contexto contemporâneo: uma revisão integrativa. In: L. F. S. S. Laurinda, \& M. C. O. Silva. (Org.). Maternidade, aborto e direitos da mulher. (1a ed., pp. 149-156). São Luís, MA: Editora Expressão Feminista.

Soares, A. N., Gonçalves, F. T. D., Melo, K. C., Da Silva, W. C., Da Silva, C. O., Hernandes, L. F., \& Alves, T. D. S. (2021). O trabalho de luto e o trabalho com o luto: percepção de estagiários de enfermagem sobre a morte e o morrer junto a pacientes hospitalizados. Research, Society and Development, 10(2), e7710212023.

Torres, A. R. A., Chagas, M. I. O., Moreira, A. C. A., Barreto, I. C. D. H. C., \& Rodrigues, E. M. (2011). O adoecimento no trabalho: repercussões na vida do trabalhador e de sua família. SANAR, 1(10), 42-48. 